\title{
Evaluation of Treatment Outcome and Acute Toxicity in Patients Undergoing Adjuvant Therapy in Ductal Carcinoma Pancreas: A Prospective Observational Study
}

\begin{abstract}
Authors:

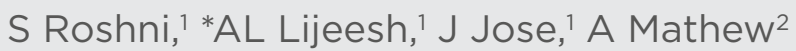

1. Department of Radiation Oncology, Regional Cancer Centre, Trivandrum, Kerala, India

2. Cancer Epidemiology and Biostatistics, Regional Cancer Centre, Trivandrum, India

*Correspondence to drlijeeshrcc@gmail.com

Disclosure: $\quad$ The authors have declared no conflicts of interest.

Received: $\quad 06.02 .21$

Accepted: $\quad 20.09 .21$

Keywords: $\quad$ Adjuvant treatment, ductal adenocarcinoma of the pancreas, pancreatic adenocarcinoma, postoperative chemoradiation.

Citation:

EMJ Oncol. 2021;9[1]:72-80.

\section{Abstract}

Ductal adenocarcinoma of the pancreas is one of the commonly diagnosed cancers and is a leading cause of cancer mortality in the population. The prognosis of patients even after undergoing a complete resection is generally poor, with a median survival of 13-20 months and a 3-year survival of $30 \%$. Therefore, adjuvant therapies including adjuvant chemoradiation and adjuvant chemotherapy are given in an effort to improve survival. In the authors' centre, all patients undergoing resection are given adjuvant chemoradiation followed by adjuvant chemotherapy. This study was conducted to evaluate the acute toxicity and treatment outcome (patterns of failure, overall and disease-free survival) of patients undergoing adjuvant therapy in resected carcinoma pancreas. Adjuvant chemoradiation was well tolerated by most patients with resected carcinoma pancreas and all patients completed chemoradiation. Adjuvant chemotherapy was associated with high haematological toxicity, similar to previously published literature. However, treatment interruptions were higher and only $77 \%$ patients completed adjuvant chemotherapy. The adjuvant gemcitabine, given on Days 1, 8, and 15, for a 4-weekly schedule was poorly tolerated by the authors' patient population and there were only fewer interruptions in patients who were switched to the 3-weekly schedule. Inclusion of a greater number of patients and longer follow-up of this study is required to clearly assess the patterns of failure and survival outcomes.
\end{abstract}

\section{INTRODUCTION}

Pancreatic cancer is the eleventh most commonly diagnosed cancer worldwide and is the seventh leading cause of cancer-related death.' It can arise from both exocrine (95\%) and endocrine

portion (5\%) of the pancreatic gland. ${ }^{2}$ The most common histology is ductal adenocarcinoma of the pancreas, which accounts for around $80 \%$ of all pancreatic cancers, ${ }^{3}$ while $65 \%$ of the cases arise in the pancreatic head, $15 \%$ in the body or tail, and $20 \%$ involve the gland diffusely. ${ }^{4}$ 
Known risk factors for development of carcinoma of the pancreas include family history, advancing age, smoking, alcoholism, obesity, diabetes mellitus (DM), and chronic calcific pancreatitis (CCP). However, age is the major determinant of pancreatic cancer. Most patients are diagnosed at $>50$ years of age, with peak incidence in the seventh and eighth decades of life. ${ }^{5}$

In terms of preventable risk factors, tobacco smoking is the most important and most studied risk factor. Individuals who smoke have a 2-3-fold higher risk of developing pancreatic cancer than people who do not smoke. A doserisk relationship has been noted as having a favourable effect of smoking cessation. ${ }^{6}$

DM is both a risk factor for disease and a consequence of early-stage pancreatic cancer. Long-term DM approximately doubles the risk of pancreatic cancer. ${ }^{7}$ However, DM can also be caused by pancreatic cancer (Type 3c DM) and, accordingly, new-onset DM can be the first clue to the diagnosis of pancreatic cancer in elderly patients. ${ }^{8}$

Of the patients with pancreatic cancer, $10 \%$ have a family history of the disease. ${ }^{9}$

The primary curative option for carcinoma of the pancreas is surgical resection but only $15-20 \%$ of patients present with a potentially resectable disease at the time of diagnosis. Local unresectability is often due to major vascular invasion. Based on the extent of vascular invasion, they are broadly classified as operable, borderline operable, or unresectable disease.

The prognosis of patients with carcinoma of the pancreas is generally poor, even for those undergoing a complete ( $\mathrm{RO}$ ) resection. Longterm survival of patients undergoing resection of localised pancreatic carcinoma is only $20 \%$, with a median survival of 13-20 months. ${ }^{10}$ Recent data suggest that the survival of patients who undergo resection of their pancreatic cancer may be improving, with a 3-year survival rate around $30 \%{ }^{11}$ and 5-year survival around $10 \% .^{12}$ In an effort to reduce recurrence rates and improve the survival of patients who have undergone resection, adjuvant therapies including chemotherapy and chemoradiation therapy have been explored.
Although adjuvant chemotherapy has been associated with an improvement in overall survival (OS), the benefits of radiotherapy remain controversial due to the conflicting results from various randomised controlled trials across the world. Adjuvant chemotherapy alone is the standard of care in Europe, based on ESPAC-1, CONKO-001, and EORTC trials. On the other hand, the American approach more often includes chemoradiotherapy in addition to adjuvant chemotherapy, based on the survival benefit from chemoradiotherapy in the GITSG study.

Apart from the ESPAC-1 trial, with its many flaws associated with the study design, no other Phase III studies have evaluated the relative benefits of chemoradiotherapy over chemotherapy alone. Hence, the standard adjuvant therapy (chemoradiation followed by chemotherapy, or chemotherapy alone) is unanswered. Data from the retrospective series and some Phase II studies suggest that patients at high-risk of recurrence may benefit from adjuvant radiation in addition to chemotherapy. The European Society for Medical Oncology (ESMO) guidelines do not recommend the use of adjuvant chemoradiation outside the context of a clinical trial. ${ }^{13}$ However, the current National Comprehensive Cancer Network (NCCN) guidelines recommend chemotherapy alone, induction chemotherapy followed by chemoradiation +/- subsequent chemotherapy, or to enrol patients in a clinical trial (all of which are Category 1 recommendations). ${ }^{14}$

In the authors' centre, all patients who underwent curative resection of exocrine pancreatic cancer were treated with adjuvant chemoradiation: 45 Gy in 25 fractions of 3D conformal radiotherapy or intensity modulated radiotherapy 5 days per week, with concurrent chemotherapy 825 $\mathrm{mg} / \mathrm{m}^{2}$ of capecitabine taken orally twice daily on all days of radiation, followed by adjuvant chemotherapy with gemcitabine $1,000 \mathrm{mg} / \mathrm{m}^{2}$ intravenously given on Days 1,8 , and 15 every 4 weeks (Q4 weekly) for 4 cycles. Patients who could not tolerate a Q4 weekly regimen were changed to Days 1 and 8 Q3 weekly schedule.

This study was conducted to evaluate the compliance, acute toxicity, and treatment outcome of patients with resected ductal adenocarcinoma of the pancreas, who were undergoing adjuvant therapy in the authors' centre. 


\section{METHODS}

Fifteen patients with ductal adenocarcinoma of the pancreas who were registered at the authors' centre and had received adjuvant treatment after surgery (chemoradiation: 45 Gy in 25 fractions with concurrent capecitabine, followed by adjuvant chemotherapy with gemcitabine given intravenously $\left[1,000 \mathrm{mg} / \mathrm{m}^{2}\right.$ on Days 1,8 , and 15 Q4 weekly for 4 cycles, or on Days 1 and 8 Q3 weekly for those not tolerating the Q4 weekly regimen) from January 2016 to June 2017 were prospectively observed for acute toxicity, relapse pattern, and survival outcomes.

\section{Inclusion Criteria of the Study}

> Histologically proven pancreatic ductal adenocarcinoma after a complete resection

> An Eastern Cooperative Oncology Group (ECOG) performance status of O-2

> Adequate haematological, hepatic, and renal function

\section{Exclusion Criteria of the Study}

> Periampullary carcinomas not arising from the pancreatic ductal epithelium

> Metastatic or locally advanced carcinoma in the pancreas and borderline resectable tumours that received neoadjuvant treatment prior to resection

$>$ Incomplete resection

\section{Treatment Protocol}

> Adjuvant therapy after resection was started as soon as possible, usually 4-6 weeks after surgery.

> Routine CT simulation was completed for radiation planning and then target volumes and organs at risk were contoured according to the Radiation Therapy Oncology Group's (RTOG) contouring guidelines.

> Radiation therapy was given using a 3D conformal radiotherapy or intensity modulated radiotherapy technique, delivering $45 \mathrm{~Gy}$ in 25 fractions (1.8 Gy per fraction), along with $825 \mathrm{mg} / \mathrm{m}^{2}$ of capecitabine taken orally twice daily for 5 days per week, until completion of radiation therapy.

> This was followed by 4 cycles of adjuvant chemotherapy with gemcitabine given by injection of $1,000 \mathrm{mg} / \mathrm{m}^{2}$ on Days 1, 8, and 15
Q4 weekly (as per RTOG 9704 protocol), or on Days 1 and 8 Q3 weekly for 6 cycles in those not tolerating Q4 weekly schedule.

\section{Outcome Measurement}

Acute toxicity was evaluated weekly during chemoradiation using the Common Terminology Criteria for Adverse Events (CTCAE) v4.0, and for 3 weeks after completion of chemoradiation and then during each cycle of adjuvant chemotherapy.

Relapse-free time was calculated from the date of registration to the date of death or first relapse. OS time was calculated from the date of diagnosis to the date of death or last followup. Disease-free survival (DFS) and OS were calculated using the Kaplan-Meier method.

The statistical significance of prognostic factors was assessed using the log-rank test (univariate analysis) and the Cox-proportional hazards regression model (multivariate analysis).

\section{RESULTS}

Fifteen eligible patients with ductal adenocarcinoma of the pancreas who were registered at the authors' centre and had received adjuvant chemoradiation and chemotherapy treatment after surgical resection, from January 2016 to June 2017, were included in the study. The sample size was small, as patients with ductal adenocarcinoma of the pancreas were the only patients included in the study. In the authors' study, periampullary carcinomas were excluded as they have an entirely different natural history and are associated with a better prognosis compared to pancreatic ductal adenocarcinomas.

\section{Patient Characteristics}

The median age of the study population was 63 years (range: 45-75 years) and the majority (53.3\%) of them were between 61 years and 70 years, which is similar to the reported literature. ${ }^{5}$ The majority (60\%) of patients were females. In this study population, $26.7 \%$ of patients were smokers. The proportion of carcinoma of the pancreas cases attributable to tobacco smoking has been estimated to be $15-30 \%$ in various study populations. ${ }^{15}$ Only $26.7 \%$ had history of alcoholism. None of the patients in study population were obese, although obesity is described as a risk factor for the development of 
carcinoma of the pancreas. ${ }^{16}$ Of all the patients in this study, $46.7 \%$ had a history of Type 2 DM and, among them, nearly half had a recent onset DM. This is similar to the reported literature that shows a high (40\%) prevalence of DM in patients with pancreatic cancer. According to the literature, $50 \%$ of people with pancreatic ductal adenocarcinoma have a history of recent onset DM. ${ }^{8}$ In the authors' study, it is more or less the same, with $42.8 \%$ of patients having DM. In the authors' study $26.7 \%$ of patients had history of CCP. However, only one patient (6.7\%) had a family history of carcinoma of the pancreas, while the proportion of patients with a positive family history is $10 \%$, according to the available published literature. $^{9}$

\section{Tumour Characteristics}

Similar to the known pattern of tumour origin, with $60-70 \%$ arising from the pancreatic head and less than $15 \%$ from body or tail, the authors also observed that $80 \%$ of their patients had tumours confined to head and $20 \%$ had tumours arising from body or tail of pancreas. The majority of patients were Stage II (40\% were Stage IIA [T3NO] and 40\% were Stage IIB [T1-3N1]). Only $13.3 \%$ were Stage III (T4, any N).

However, $40 \%$ of patients had inadequate nodal sampling, which is defined as less than 15 nodes removed during surgery, as per the detailed pathology report. Forty percent of tumours were positive for perineural invasion, which was less than the published literatures, showing a high incidence of perineural invasion of around 70-100\% in pancreatic ductal adenocarcinoma. ${ }^{17}$ Only $53.3 \%$ of patients had preoperative carbohydrate antigen (CA) 19-9 level values available as the majority of patients (66.6\%) had surgery at another centre and reported to the authors' hospital for adjuvant treatment. The normal range of CA $19-9$ is $0-37 \mathrm{U} / \mathrm{mL}$. Two patients (13.3\%) had preoperative values of more than $500 \mathrm{U} / \mathrm{mL}$ and $20 \%$ had values between $100-500 \mathrm{U} / \mathrm{mL}$. Post-operative CA 19-9 values were available for all patients and the majority (73.3\%) had values below $50 \mathrm{U} / \mathrm{mL}$.

\section{Treatment Characteristics}

However, only three patients (23\%) completed all 12 doses of adjuvant chemotherapy without any interruptions. The rest (77\%) had interruptions in form of a delay, skipped cycles, or dose reduction. Of these, $46.0 \%$ of patients had a delay in chemotherapy, $69.0 \%$ of patients had at least one chemotherapy doses skipped, and $61.6 \%$ of patients required dose reduction. Haematological toxicity accounted for delay in $66 \%$ of cases, for skipping chemotherapy in $69 \%$ of case and a dose reduction in $75 \%$ of cases.

The chemotherapy schedule was changed from an injection of gemcitabine on Days 1, 8, and 15 Q4 weekly to Days 1 and 8 Q3 weekly due to poor tolerance in 4 patients (31\%).

Chemotherapy regimen was changed from an injection of gemcitabine to an injection of 5-flurouracil plus an injection of calcium leucovorin in one patient $(7.7 \%)$, due to repeated Grade 3 liver function test alteration.

Chemotherapy was stopped in two patients due to poor general condition and one patient developed systemic metastasis before the completion of adjuvant chemotherapy. In total, only $77 \%$ completed adjuvant chemotherapy (54\% completed with interruption and 23\% without any interruptions). This, however, was hugely different from the RTOG 9704 trial,, 1 where $90 \%$ of patients completed chemotherapy in the gemcitabine arm. This might be due the inclusion of patients with good performance and nutritional status in the trial setting, which was not possible in the authors' scenario. Their patients came from low socio-economic status and the majority (80\%) had a BMI less than 25 , which is hugely different from a western population. However, in the GERCOR Phase II study, only $73.3 \%$ patients completed adjuvant chemotherapy, similar to the authors' study. ${ }^{18}$

\section{Acute Toxicity}

Table 1 shows toxicity during chemoradiation, and Table 2 shows toxicity during adjuvant chemotherapy. After a median follow-up of 12.5 months, three patients had a recurrence (20.0\%). The median and mean times to relapse were 2 and 2.5 months, respectively. One patient had a local recurrence (33.3\%), and two patients had a systemic recurrence (66.6\%).

This is similar to the pattern of recurrence observed in the RTOG 9704 trial, where the incidence in loco-regional relapse was 30\% and systemic relapse was 70\%. Relapse was identified by an asymptomatic marker rise (CA 19-9) alone 
in the patient with local recurrence and he was salvaged successfully and is alive and disease-free. In one patient with systemic relapse, the marker rise preceded the development of symptoms and radiological evidence of relapse. Hence, CA 19-9 monitoring should be a part of surveillance during follow-up after adjuvant treatment. Median OS and DFS were 15 and 14 months, respectively. OS and DFS at 18 months were $65.5 \%$ and $71.6 \%$, respectively (Figure 1 ). The presence of CCP was associated with a significant difference in OS in both univariate and multivariate analysis.

\section{Table 1: Toxicity during chemoradiation.}

\begin{tabular}{|c|c|c|}
\hline & Frequency & Percentage (\%) \\
\hline \multicolumn{3}{|c|}{ ECOG PS } \\
\hline 1 & 12 & 80.0 \\
\hline 2 & 3 & 20.0 \\
\hline 3 & 0 & 0.0 \\
\hline 4 & 0 & 0.0 \\
\hline \multicolumn{3}{|c|}{ Weight loss } \\
\hline Grade 1 & 2 & 13.0 \\
\hline Grade 2 & 0 & 0.0 \\
\hline Grade 3 & 0 & 0.0 \\
\hline \multicolumn{3}{|c|}{ Weight loss } \\
\hline Grade 1 & 15 & 100.0 \\
\hline Grade 2 & 4 & 26.6 \\
\hline Grade 3 & 1 & 6.7 \\
\hline \multicolumn{3}{|l|}{ Nausea } \\
\hline Grade 1 & 15 & 100.0 \\
\hline Grade 2 & 7 & 46.6 \\
\hline \multicolumn{3}{|c|}{ Vomiting } \\
\hline Grade 1 & 6 & 40.0 \\
\hline Grade 2 & 2 & 13.3 \\
\hline Grade 3 & 1 & 6.7 \\
\hline Grade 4 & 0 & 0.0 \\
\hline \multicolumn{3}{|c|}{ Diarrhoea } \\
\hline Grade 1 & 2 & 13.3 \\
\hline Grade 2 & 0 & 0.0 \\
\hline Grade 3 & 0 & 0.0 \\
\hline Grade 4 & 0 & 0.0 \\
\hline \multicolumn{3}{|c|}{ Abdominal pain } \\
\hline Grade 1 & 7 & 46.6 \\
\hline Grade 2 & 1 & 6.7 \\
\hline
\end{tabular}


Table 1 contined.

\begin{tabular}{|c|c|c|}
\hline & Frequency & Percentage (\%) \\
\hline Grade 3 & 0 & 0.0 \\
\hline \multicolumn{3}{|l|}{ Anaemia } \\
\hline Grade 1 & 1 & 13.3 \\
\hline Grade 2 & 0 & 0.0 \\
\hline Grade 3 & 0 & 0.0 \\
\hline Grade 4 & 0 & 0.0 \\
\hline \multicolumn{3}{|c|}{ Neutropenia } \\
\hline Grade 1 & 2 & 13.3 \\
\hline Grade 2 & 0 & 0.0 \\
\hline Grade 3 & 0 & 0.0 \\
\hline Grade 4 & 0 & 0.0 \\
\hline \multicolumn{3}{|c|}{ Thrombocytopenia } \\
\hline Grade 1 & 1 & 6.7 \\
\hline Grade 2 & 1 & 6.7 \\
\hline Grade 3 & 0 & 0.0 \\
\hline Grade 4 & 0 & 0.0 \\
\hline \multicolumn{3}{|c|}{ Hypoalbuminaemia } \\
\hline Grade 1 & 2 & 13.3 \\
\hline Grade 2 & 1 & 6.7 \\
\hline Grade 3 & 0 & 0.0 \\
\hline Grade 4 & 0 & 0.0 \\
\hline
\end{tabular}

ECOG PS: Eastern Cooperative Oncology Group performance status.

Table 2: Toxicity during adjuvant chemotherapy.

\begin{tabular}{|c|c|c|}
\hline & Frequency & Percentage (\%) \\
\hline \multicolumn{3}{|c|}{ ECOG PS } \\
\hline 1 & 8 & 75 \\
\hline 2 & 5 & 38 \\
\hline 3 & 2 & 13.3 \\
\hline 4 & 0 & 0.0 \\
\hline \multicolumn{3}{|c|}{ Weight loss } \\
\hline Grade 1 & 13 & 100 \\
\hline Grade 2 & 7 & 53.8 \\
\hline Grade 3 & 2 & 15.3 \\
\hline
\end{tabular}


Table 2 continued.

\begin{tabular}{|c|c|c|}
\hline & Frequency & Percentage (\%) \\
\hline \multicolumn{3}{|l|}{ Nausea } \\
\hline Grade 1 & 13 & 100.0 \\
\hline Grade 2 & 6 & 46.2 \\
\hline Grade 3 & 1 & 7.7 \\
\hline \multicolumn{3}{|l|}{ Vomiting } \\
\hline Grade 1 & 4 & 30.1 \\
\hline Grade 2 & 2 & 15.3 \\
\hline Grade 3 & 1 & 7.7 \\
\hline Grade 4 & 0 & 0.0 \\
\hline \multicolumn{3}{|c|}{ Abdominal pain } \\
\hline Grade 1 & 2 & 15.3 \\
\hline Grade 2 & 1 & 7.7 \\
\hline Grade 3 & 1 & 7.7 \\
\hline \multicolumn{3}{|l|}{ Anaemia } \\
\hline Grade 1 & 4 & 30.1 \\
\hline Grade 2 & 3 & 23.1 \\
\hline Grade 3 & 3 & 23.1 \\
\hline Grade 4 & 0 & 0.0 \\
\hline \multicolumn{3}{|c|}{ Neutropenia } \\
\hline Grade 1 & 3 & 23.1 \\
\hline Grade 2 & 2 & 15.3 \\
\hline Grade 3 & 6 & 46.2 \\
\hline Grade 4 & 2 & 15.3 \\
\hline \multicolumn{3}{|c|}{ Thrombocytopenia } \\
\hline Grade 1 & 6 & 46.2 \\
\hline Grade 2 & 1 & 7.7 \\
\hline Grade 3 & 0 & 0.0 \\
\hline Grade 4 & 1 & 7.7 \\
\hline \multicolumn{3}{|c|}{ Worsened bilirubin } \\
\hline Grade 1 & 1 & 7.7 \\
\hline Grade 2 & 1 & 7.7 \\
\hline Grade 3 & 0 & 0.0 \\
\hline Grade 4 & 0 & 0.0 \\
\hline \multicolumn{3}{|c|}{ Worsened SGOT/SGPT } \\
\hline Grade 1 & 2 & 15.3 \\
\hline Grade 2 & 2 & 15.3 \\
\hline
\end{tabular}

ECOG PS: Eastern Cooperative Oncology Group performance status; SGOT: serum glutamic-oxaloacetic transaminase; SGPT: serum glutamic-pyruvic transaminase. 
Table 2 continued.

\begin{tabular}{|c|c|c|}
\hline & Frequency & Percentage (\%) \\
\hline Grade 3 & 2 & 15.3 \\
\hline Grade 4 & 0 & 0 \\
\hline \multicolumn{3}{|c|}{ Hypoalbuminaemia } \\
\hline Grade 1 & 5 & 38.5 \\
\hline Grade 2 & 4 & 30.1 \\
\hline Grade 3 & 1 & 7.7 \\
\hline Grade 4 & 1 & 7.7 \\
\hline
\end{tabular}

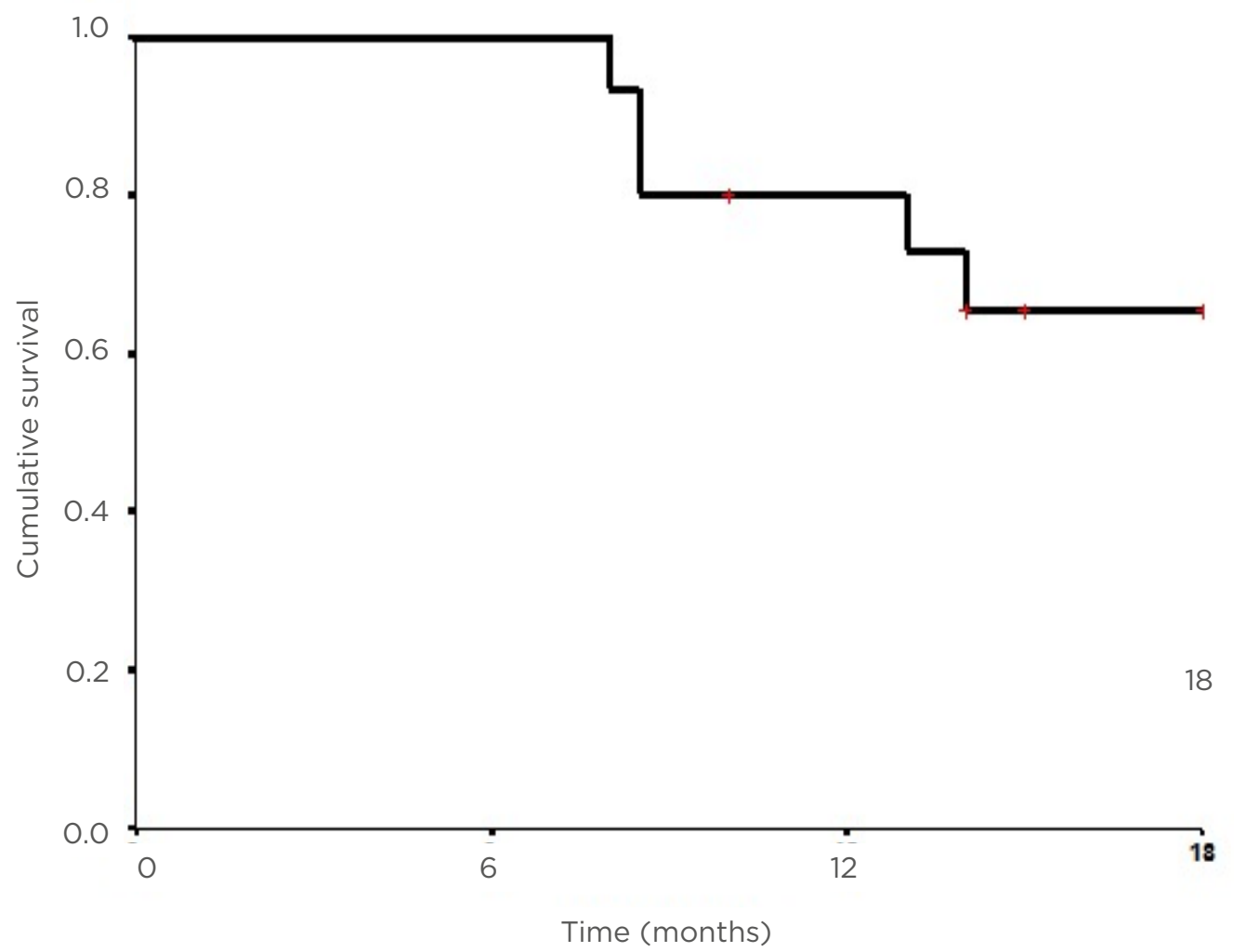

Figure 1: Kaplan-Meier curve showing the overall survival for the study population.

\section{DISCUSSION}

The data demonstrates that locally advanced pancreatic adenocarcinoma treated with adjuvant chemoradiation was well tolerated by most patients (with only one reported Grade 3 toxicity, being nausea, vomiting, fatigue, and no haematological toxicity more than Grade 2). All patients completed chemoradiation (with interruption in only 13.3). OS at 12 months was $80 \%$ and at 18 months was $65.5 \%$. The median OS was 15 months. In the RTOG 9704 trial, the OS at 12 months and 18 months were $70 \%$ and 55\%, respectively. The relatively high OS in this study compared to the RTOG trial might be due to the smaller sample size of the study. 
Compared with chemoradiation, adjuvant chemotherapy was associated with a high incidence of haematological toxicity (Grade 3 or higher neutropoenia in $61.5 \%$ of cases and Grade 4 neutropoenia alone in $15.3 \%$ of cases) similar to the RTOG 9704 trial (the followed protocol in this study). However, treatment interruptions were higher compared to the RTOG trial and only $77 \%$ completed adjuvant chemotherapy with interruptions in $54 \%$. The main cause of interruption was haematological toxicity.

The chemotherapy schedule was changed from an injection of gemcitabine on Days 1, 8, and 15 Q4 weekly to Days 1 and 8 Q3 weekly due to poor tolerance in 4 patients (31\%). There were fewer interruptions in patients who were changed to the 3-weekly schedule.

\section{CONCLUSION}

Adjuvant chemoradiation was well tolerated by the majority of patients. Adjuvant chemotherapy was associated with a high incidence of haematological toxicity.

The ongoing RTOG 0848 trial is evaluating the approach of deferring chemoradiation until the completion of adjuvant chemotherapy as an option to decrease the added bone marrow toxicity of radiation that could lead on to chemotherapy interruptions due to haematological toxicities. However, until the results of this trial are available, no such recommendations can be made as if now.

This warrants the need for similar studies with inclusion of greater number of patients and longer follow-up period.

\section{References}

1. Ferlay $\mathrm{J}$ et al. Cancer Incidence and Mortality Worldwide: IARC CancerBase No. 10 Lyon. France: International Agency for Research on Cancer. 2010. GLOBOCAN 2008.

2. Cowgill SM, Muscarella P. The genetics of pancreatic cancer. Am J Surg. 2003;186(3):279-86.

3. Wilentz RE, Hruban RH. Pathology of cancer of the pancreas. Surg Oncol Clin N Am. 1998;7(1):43-65.

4. Esposito I et al. Pathology of pancreatic ductal adenocarcinoma: facts, challenges and future developments. World J Gastroenterol. 2014;20(38):13833-41.

5. Bosetti $C$ et al. Pancreatic cancer: overview of descriptive epidemiology. Mol Carcinog. 2012;51(1):3-13.

6. Bosetti $C$ et al. Cigarette smoking and pancreatic cancer: an analysis from the International Pancreatic Cancer Case-Control Consortium (Panc4). Ann Oncol. 2011;23(7):1880-8.

7. Bosetti $C$ et al. Diabetes, antidiabetic medications, and pancreatic cancer risk: an analysis from the
International Pancreatic Cancer Case-Control Consortium. Ann Oncol. 2014;25(10):2065-72.

8. Chari ST et al. Pancreatic cancer-associated diabetes mellitus: prevalence and temporal association with diagnosis of cancer. Gastroenterology. 2008;134(1):95-101.

9. Turati $\mathrm{F}$ et al. Family history of cancer and the risk of cancer: a network of case-control studies. Ann Oncol. 2013;24(10):2651-6.

10. Geer RJ, Brennan MF. Prognostic indicators for survival after resection of pancreatic adenocarcinoma. Am J Surg. 1993;165(1):68-73.

11. Lillemoe KD. Current management of pancreatic carcinoma. Ann Surg. 1995;221(2):133-48.

12. Wagner $M$ et al. Curative resection is the single most important factor determining outcome in patients with pancreatic adenocarcinoma. $\mathrm{Br} \mathrm{J}$ Surg. 2004;91(5):586-94.

13. Ducreux $M$ et al. Cancer of the pancreas: ESMO Clinical Practice Guidelines for diagnosis, treatment and follow-up. Ann Oncol. 2015;26(Suppl 5):v56-68.

14. Tempero MA et al. Pancreatic adenocarcinoma, version 2.2017, NCCN clinical practice guidelines in oncology. J Natl Compr Canc Netw. 2017;15(8):1028-61.

15. Parkin DM. 2. Tobacco-attributable cancer burden in the UK in 2010. Br J Cancer. 2011;105(Suppl 2):S6-13.

16. Genkinger JM et al. Central adiposity, obesity during early adulthood, and pancreatic cancer mortality in a pooled analysis of cohort studies. Ann Oncol. 2015;26(11):2257-66

17. Lowenfels AB, Maisonneuve $P$. Epidemiology and risk factors for pancreatic cancer. Best Pract Res Clin Gastroenterol. 2006;20(2):197-209.

18. Van Laethem JL et al. Adjuvant gemcitabine alone versus gemcitabine-based chemoradiotherapy after curative resection for pancreatic cancer: a randomized EORTC-40013-22012/ FFCD-9203/GERCOR Phase II study. J Clin Oncol. 2010;28(29):4450-6. 